\title{
X-linked inhibitor of apoptosis deficiency in the TRAMP mouse prostate cancer model
}

\author{
C Hwang ${ }^{1,5}$, KA Oetjen $^{2,5}$, D Kosoff ${ }^{2}$, KJ Wojno ${ }^{3}$, MA Albertelli ${ }^{4}$, RL Dunn ${ }^{3}$, DM Robins ${ }^{4}$, KA Cooney ${ }^{1,3}$ and CS Duckett ${ }^{, 1,2}$
}

Deregulation of apoptotic pathways plays a central role in cancer pathogenesis. X-linked inhibitor of apoptosis protein (XIAP), is an antiapoptotic molecule, whose elevated expression has been observed in tumor specimens from patients with prostate carcinoma. Studies in human cancer cell culture models and xenograft tumor models have demonstrated that loss of XIAP sensitizes cancer cells to apoptotic stimuli and abrogates tumor growth. In view of these findings, XIAP represents an attractive antiapoptotic therapeutic target for prostate cancer. To examine the role of XIAP in an immunocompetent mouse cancer model, we have generated transgenic adenocarcinoma of the mouse prostate (TRAMP) mice that lack XIAP. We did not observe a protective effect of Xiap deficiency in TRAMP mice as measured by tumor onset and overall survival. In fact, there was an unexpected trend toward more aggressive disease in the Xiap-deficient mice. These findings suggest that alternative mechanisms of apoptosis resistance are playing a significant oncogenic role in the setting of Xiap deficiency. Our study has implications for XIAP-targeting therapies currently in development. Greater understanding of these mechanisms will aid in combating resistance to XIAP-targeting treatment, in addition to optimizing selection of patients who are most likely to respond to such treatment.

Cell Death and Differentiation (2008) 15, 831-840; doi:10.1038/cdd.2008.15; published online 8 February 2008

Apoptosis is a process of cell death that is tightly regulated by a cadre of both pro- and antiapoptotic proteins. In contrast to healthy cells, a hallmark of cancerous cells is the acquired capacity to evade this process of programmed cell death. ${ }^{1,2}$ The acquisition of genetic lesions leading to oncogene activation normally triggers a program of apoptosis or senescence. Additionally, the tumor microenvironment often exposes malignant cells to apoptotic stimuli, such as hypoxia or activation of death receptors. Thus, suppression of the pathway leading to cell death has been suggested as a necessarily early event in the development of neoplasia.

Execution of the apoptotic cell death process is carried out by caspases, a family of cysteine aspartate proteases. ${ }^{3,4}$ During apoptosis, loss of mitochondrial integrity or engagement of death receptors leads to the activation of initiator caspase-9 or -8, respectively. In either case, the initiator caspases cleave and activate effector caspases, including caspase-3 or -7 . The cascade of caspase cleavage is regulated by $X$-linked inhibitor of apoptosis protein (XIAP). XIAP belongs to the IAP family, characterized by containing at least one zinc-binding baculovirus IAP repeat. ${ }^{5}$ The only member of the IAP family that potently inhibits caspase activity, XIAP has been demonstrated to directly inhibit caspases-3, -7 and -9 , blocking both intrinsic and extrinsic apoptotic signals. ${ }^{6}$ Given its role in apoptosis, there has been much interest in understanding the role of XIAP in cancer and evaluating XIAP as a therapeutic target. ${ }^{7-9}$

XIAP overexpression has been reported in a variety of human cancers. ${ }^{10-16}$ Increased XIAP levels have been linked to escaping anoikis and apoptosis induced by radiation, chemotherapy and death receptor ligands. ${ }^{13,17-22}$ Furthermore, antagonism of XIAP has been reported to have antitumor activity in a number of models, including prostate cancer. ${ }^{21,23-25}$ Consistent with an antiapoptotic role, high levels of XIAP have an adverse prognosis in certain cancers. ${ }^{14,15}$ However, there was an unexpected favorable prognosis seen in prostate and non-small cell lung cancers with high levels of XIAP expression. ${ }^{12,26}$

We thus chose to further examine the role of XIAP in a tumor model that would resemble human cancer more closely than xenograft or in vitro studies. In this study, we describe an evaluation of prostate cancer development in the presence and absence of XIAP using the transgenic adenocarcinoma of the mouse prostate (TRAMP) model. Prostate-specific expression of SV40 T-antigen in TRAMP mice results in 100\% penetrance of prostate tumors and a substantial proportion with metastatic disease. ${ }^{27}$ XIAP expression is elevated in TRAMP tumors compared to normal prostate epithelium, ${ }^{12}$ further justifying an evaluation of the effect of Xiap deficiency in this model.

\footnotetext{
${ }^{1}$ Department of Internal Medicine, University of Michigan Medical School, Ann Arbor, MI, USA; ${ }^{2}$ Department of Pathology, University of Michigan Medical School, Ann Arbor, MI, USA; ${ }^{3}$ Department of Urology, University of Michigan Medical School, Ann Arbor, MI, USA and ${ }^{4}$ Department of Human Genetics, University of Michigan Medical School, Ann Arbor, MI, USA

${ }^{*}$ Corresponding author: CS Duckett, Department of Pathology and Internal Medicine, University of Michigan, BSRB, Room 2057, 109 Zina Pitcher Place, Ann Arbor, Ml 48109-2200, USA. Tel.: + 734615 6414; Fax: + 734763 2162; E-mail: colind@umich.edu

${ }^{5}$ These authors contributed equally to this work.

Keywords: XIAP; BIRC4; prostate cancer

Abbreviations: C-IAP1, cellular inhibitor of apoptosis protein 1; C-IAP2, cellular inhibitor of apoptosis protein 2; IAP, inhibitor of apoptosis protein; MRI, magnetic resonance imaging; PIN, prostatic intraepithelial neoplasia; RIAP, rodent inhibitor of apoptosis protein; TRAMP, transgenic adenocarcinoma of mouse prostate; TUNEL, terminal dUTP nick end labeling; XIAP, X-linked inhibitor of apoptosis protein

Received 12.9.07; revised 20.12.07; accepted 07.1.08; Edited by A Villunger; published online 08.2.08
} 
Surprisingly, we found no evidence for a protective effect of Xiap deficiency in TRAMP mice. Xiap-deficient mice demonstrated no difference in tumor onset or overall survival compared to controls. Furthermore, tumor histology revealed similar patterns of differentiation and frequencies of apoptosis and proliferation in tumors from both groups. To our knowledge, these experiments represent the first examination of XIAP in an immunocompetent cancer model and differ markedly from previously published studies using xenografted cell lines. These results are relevant to the development of therapeutics, currently in clinical trials, that specifically antagonize XIAP.

\section{Results}

Loss of XIAP in TRAMP mice does not prevent tumor development or improve survival. Xiap-deficient TRAMP mice were generated with the hypothesis that mice deficient in XIAP may be protected from tumor formation compared to wild-type controls. Prostate size was monitored by magnetic resonance imaging (MRI). Representative MRI images from two littermate pairs of TRAMP mice are shown in Figure 1. In the first pair, tumor onset occurred sooner in the Xiapdeficient mouse compared to its wild-type littermate, but there was no appreciable difference in tumor onset or growth in the second littermate pair. Aggregate Kaplan-Meier analysis showed no difference between the two groups of mice (data not shown). The median age of tumor onset was 25.9 weeks for Xiap-deficient mice and 25.6 weeks for wildtype mice (Xiap-deficient mice $n=9$; wild-type mice $n=8$; $P=0.88$ )

In addition to MRI, tumor onset was evaluated by abdominal palpation. The median age at the time of palpable tumor onset was 26.0 weeks in Xiap-deficient mice and 27.3 weeks in littermate controls (Xiap-deficient mice $n=13$; wild-type mice $n=11$ ). Kaplan-Meier analysis was used to compare the a

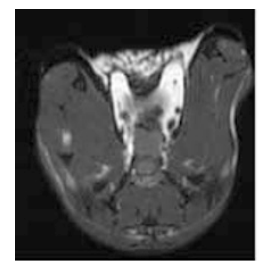

19 weeks

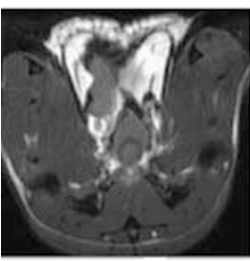

26 weeks

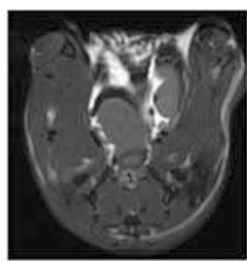

22 weeks

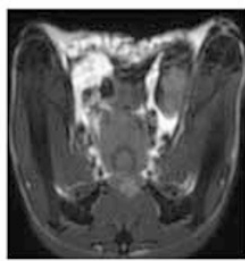

30 weeks

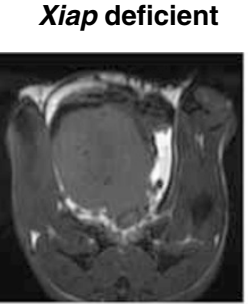

24 weeks

Xiap wild type

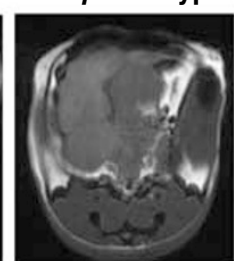

40 weeks

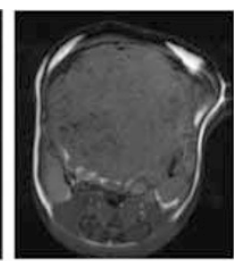

26 weeks

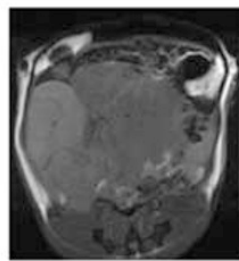

42 weeks

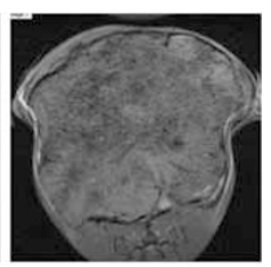

28 weeks

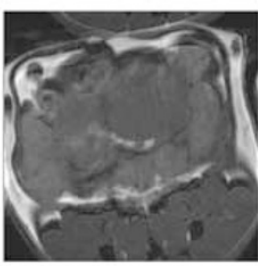

46 weeks

b

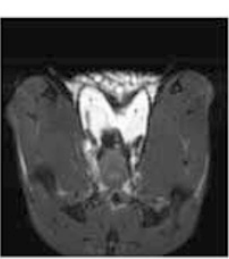

15 weeks

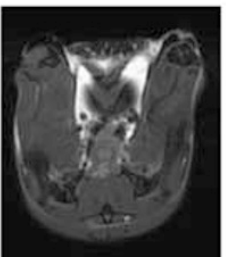

15 weeks

Xiap deficient

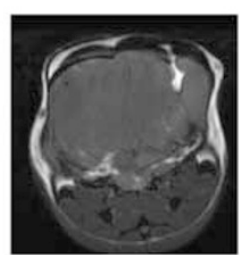

37 weeks

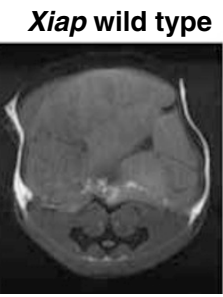

37 weeks

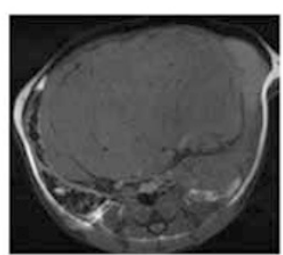

39 weeks

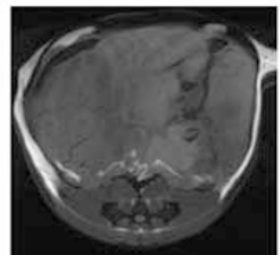

39 weeks

Figure 1 MRI images of prostate tumors in Xiap-deficient and wild-type TRAMP mice. MRI scans are shown for two representative pairs (a, b) of Xiap-deficient and wild-type TRAMP mice 
a

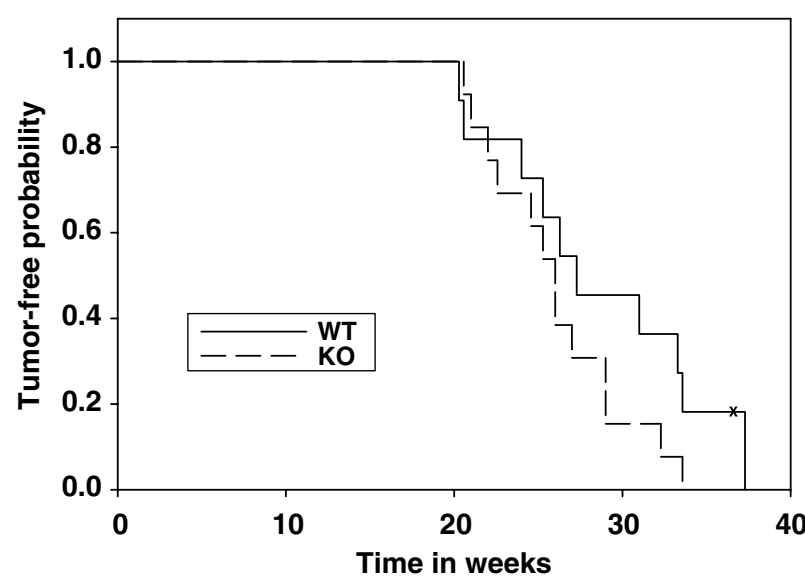

b

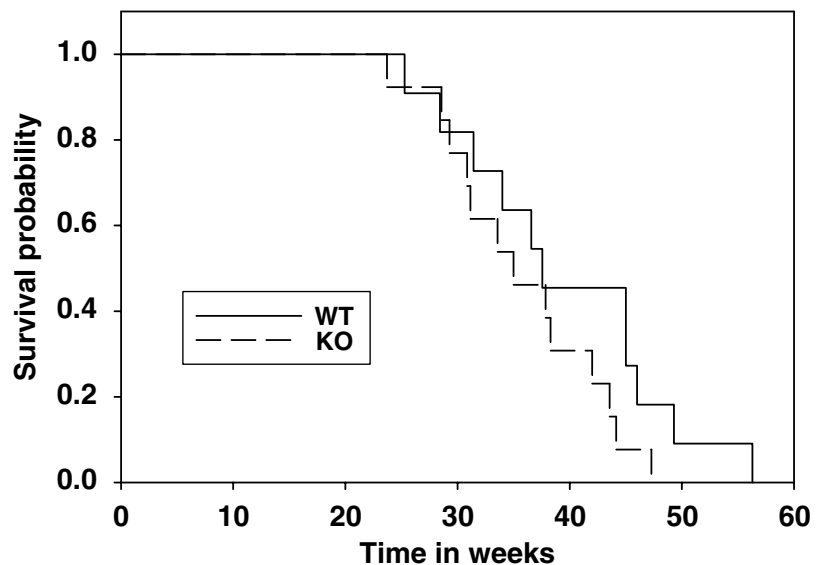

Figure 2 Kaplan-Meier analysis for tumor onset and overall survival. (a) Kaplan-Meier curves were plotted for Xiap-deficient (dotted line) and wildtype (solid line) TRAMP mice. The probability of remaining without tumor is plotted against age in weeks. One wild-type TRAMP mouse died from unknown causes at 36 weeks of age before evidence of tumor onset, and this mouse $(\mathrm{x})$ was censored. A one-sided log-rank analysis yields a $P$-value of 0.924 , indicating a $7.6 \%$ probability that Xiap-deficient mice would protect against tumor onset. (b) Kaplan-Meier curves were plotted for Xiap-deficient (dotted line) and wild-type (solid line) TRAMP mice. The probability of survival is plotted against age in weeks. A one-sided log rank analysis yields a $P$-value of 0.928 , indicating a $7.2 \%$ probability that Xiap deficiency is protective for overall survival

probability of remaining without tumor between the two groups (Figure 2a). There was no evidence for a protective effect of Xiap deficiency in TRAMP mice. In fact, there was an unexpected trend for the Xiap-deficient mice to develop tumors earlier than wild-type controls, although the difference was not statistically significant (two-sided $P$-value $=0.15$ ).

Although Xiap deficiency did not alter TRAMP tumor onset, inhibition of apoptosis by XIAP could contribute to a more lethal phenotype later in tumor progression. However, there was no evidence for a protective effect of Xiap deficiency in TRAMP mice on overall survival, and the trend for poorer outcome in the Xiap-deficient mice was again observed (Figure 2b). The median age at time of death was 35.0 weeks for Xiap-deficient TRAMP mice $(n=13)$, and the median age of control TRAMP mice at the time of death was 37.6 weeks $(n=11)$, but this difference was not statistically significant (two-sided $P$-value $=0.15$ ).

Xiap deficiency does not retard growth of TRAMP tumors. Since apoptosis may affect tumor growth without significantly impacting either tumor onset or overall survival, we hypothesized that a deficiency in XIAP may be expected to result in a decreased tumor burden. To assess tumor burden, the mass of the primary tumor at the time of necropsy was determined and tumor mass as a percentage of the mass of the mouse at necropsy was calculated. By both of these measures, Xiap deficiency did not result in a decrease in tumor burden as compared to wild-type controls (Figure 3a). Indeed, average tumor mass was somewhat greater in Xiap-deficient TRAMP mice $(11.1 \pm 5.06 \mathrm{~g})$ than littermate controls $(9.05 \pm 4.78 \mathrm{~g})$ despite the shortened survival observed above.

Although an effect on tumor burden at the time of death was not seen, tumor size may reflect the duration of growth as opposed to tumor growth rate. Thus, the time from palpable tumor to time of killing was measured. Generally, a prolonged duration between tumor onset and killing would indicate slower tumor growth. However, when Xiap-deficient TRAMP mice were compared to controls, there was no delay from the time of palpable tumor to time of killing (Figure 3b, last bars). As previously noted, the time to palpable tumor and time to death for Xiap-deficient TRAMP mice was shorter than controls.

Tumor growth was also assessed over time by clinical examination on a four-point scale. The time from palpable tumor to larger tumors was calculated and compared between Xiap-deficient TRAMP mice and controls. In agreement with our previous finding that Xiap deficiency does not delay time to killing or time from palpable tumor to killing, tumors in Xiap-deficient mice did not grow more slowly than tumors in controls (Figure $3 \mathrm{c}$ ). To the contrary, the average time from palpable tumor to larger tumors (size 3 or 4 ) was seen to be shorter in Xiap-deficient mice.

Xiap deficiency does not result in less aggressive TRAMP tumors. When tumor mass at autopsy was measured, it was observed that the mass of the primary tumor varied significantly, indicating that local growth did not always correspond to tumor lethality. Tumor aggressiveness also correlates with tumor grade and metastatic potential. Elevated expression of XIAP has been observed to correlate with higher tumor grades in renal cell carcinoma and breast cancer, ${ }^{10,14}$ although this is not the case in other cancers. ${ }^{26,28}$ To evaluate the effect of Xiap deficiency on tumor grade, tumor differentiation was assessed as another measure of tumor aggressiveness. Poorly and moderately differentiated carcinomas, as well as the more benign phylloides tumor, were observed in both Xiap-deficient and wild-type TRAMP mice. Examples of poorly differentiated and moderately differentiated primary tumors in both Xiapdeficient and wild-type mice are shown in Figure 4a. When tabulated, 67\% (6/9) Xiap-deficient mice were noted to have carcinoma and the remainder $(33 \%, 3 / 9)$ were phylloides. In comparison, $50 \%(4 / 8)$ of tumors from control mice were carcinoma and $50 \%$ (4/8) were phylloides. Among those 

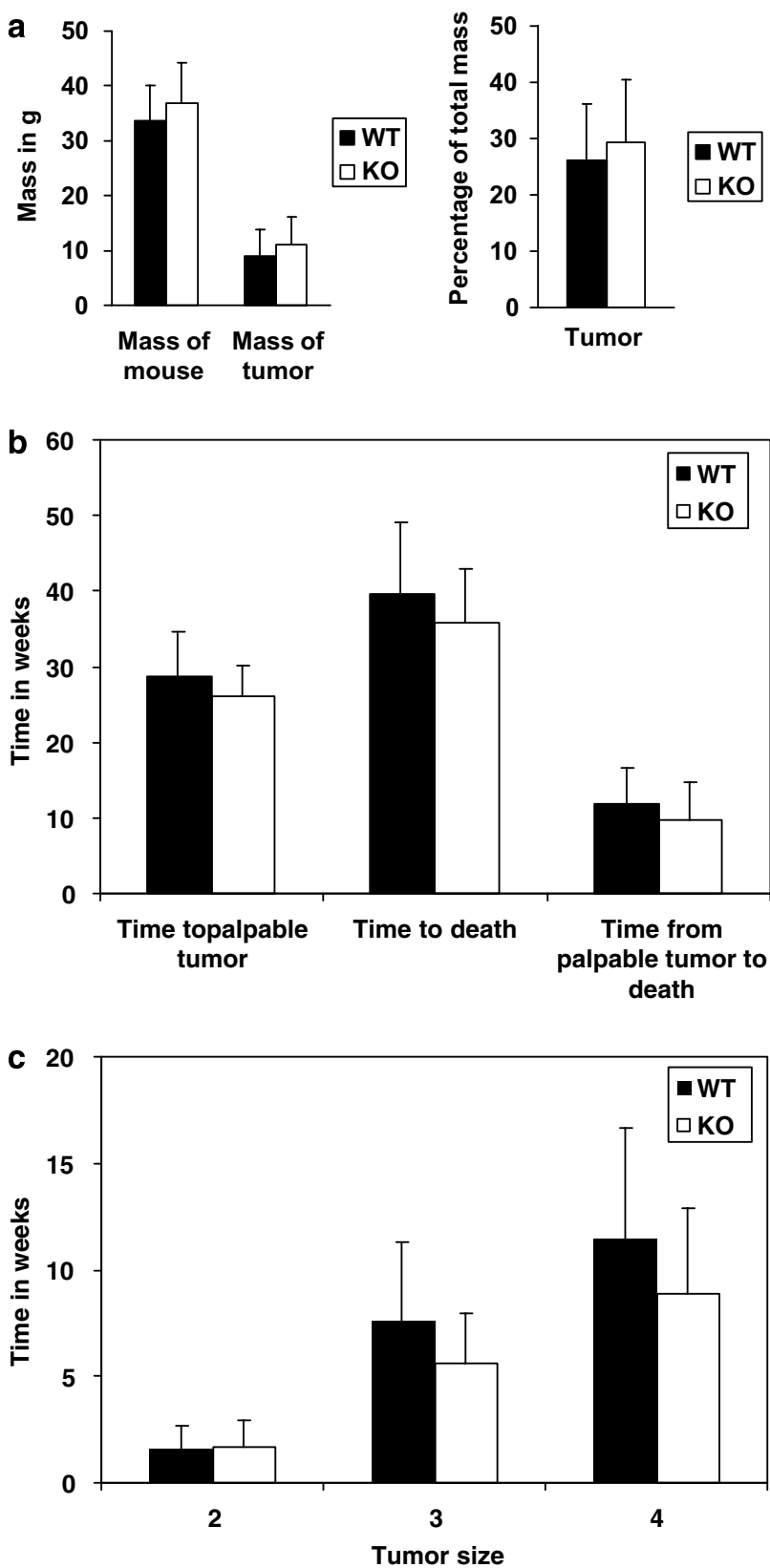

Figure 3 Comparison of tumor characteristics in Xiap-deficient and wild-type TRAMP mice. (a) Mass of animals and mass of tumors at necropsy were compared between the wild-type and Xiap-deficient mice. The tumor mass as a percentage of total mouse mass at the time of necropsy was also compared. Mean values are plotted with error bars representing one standard deviation. (b) Time to palpable tumor, time to death and time from palpable tumor to death were assessed for Xiapdeficient and wild-type mice. Mean values for each variable are plotted with error bars representing one standard deviation. (c) The time in weeks from tumor onset (size 1) to larger tumors (sizes 2-4) was calculated for each cohort of mice. Mean values for each variable are plotted with error bars representing one standard deviation

classified as carcinoma, tumors were poorly differentiated in $83 \%$ of Xiap-deficient mice (5/6 mice with carcinoma) and $50 \%$ of controls (2/4 mice with carcinoma). Overall, this equated to $56 \%$ of the entire cohort of Xiap-deficient mice with poorly differentiated carcinoma and $25 \%$ of the wild-type cohort (Figure 4b). Therefore, Xiap-deficient mice are not protected from more aggressive histologic subtypes of TRAMP tumors.

Xiap-deficient and control TRAMP mice were also evaluated for metastatic spread of tumor. An increase in XIAP has been implicated in resistance to anoikis and increased metastatic potential. ${ }^{17,20}$ However, at necropsy, both Xiapdeficient and wild-type mice were observed to have pelvic nodal metastases and distant metastases. Metastatic deposits were confirmed histologically and representative images are presented in the right-hand panels of Figure $4 \mathrm{a}$. When quantified, $56 \%$ (5/9) of Xiap-deficient TRAMP mice were noted to have metastases compared to $63 \%$ (5/8) of control TRAMP mice (Figure 4b). Evidence of distant metastases (liver and lung) was identified in three Xiap-deficient TRAMP mice (33\%, 3/9 mice) compared to two control mice (25\%, 2/8 mice). Thus, Xiap-deficient mice were not protected from metastatic spread. The majority of metastatic lesions in both Xiap-deficient and control TRAMP mice were poorly differentiated.

Xiap deficiency does not result in decreased incidence of pre-invasive lesions. Elevation of XIAP expression has been observed in pre-invasive prostatic intraepithelial neoplasia (PIN) specimens from patients treated for prostate cancer as well as TRAMP mice. ${ }^{12}$ To investigate the possibility that XIAP contributes to early tumor development, we studied the effect of loss of XIAP in a cohort of Xiap-deficient and control TRAMP mice who were followed until 25 weeks of age. Mice were assessed for prostate gland histology and micrometastatic disease (Table 1). None of the 25-week-old mice had evidence of micrometastases. There was no difference between Xiapdeficient and wild-type TRAMP mice in the incidence of PIN or microscopic carcinoma.

Loss of XIAP does not affect apoptosis or proliferation of tumor cells in vivo. Because of the accepted role of $\mathrm{XIAP}$ in cell death, we examined tumor specimens from Xiapdeficient mice by terminal dUTP nick end labeling (TUNEL) staining to assess the number of cells undergoing apoptosis (Figure 5a). There was no difference seen in TUNEL staining between the Xiap-deficient and wild-type tumors. Moreover, when quantitated, the apoptotic index was not increased in Xiap-deficient compared to wild-type TRAMP tumors (Figure $5 b$ ). In most tumors from both wild-type and Xiapdeficient TRAMP mice, the apoptotic index was on the order of 10 apoptotic cells per 1000, with only a few tumors exhibiting higher apoptotic indices. There did not seem to be a correlation between apoptotic index and histologic subtype. Of the three tumors with apoptotic indices greater than 15 cells per 1000 , one was poorly differentiated, one was well differentiated and one was phylloides.

In view of previous work in resected lung cancer suggesting an increased proliferative and mitotic index in tumors with low XIAP expression, ${ }^{26}$ we also performed an analysis of the proliferative rates present in these TRAMP tumors. Using both nuclear Ki-67 staining and mitotic figures as markers for proliferation, we did not observe any differences between the wild-type and Xiap-deficient TRAMP tumors (Figure 6). 
a
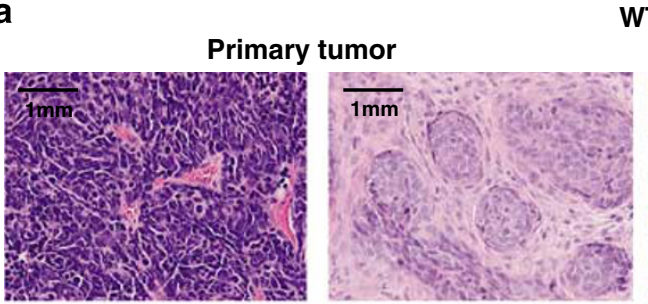

WT

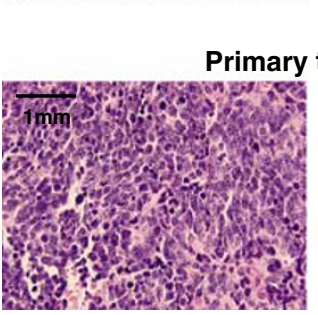

tumor
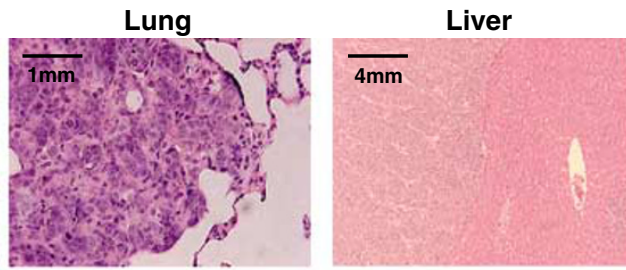

KO
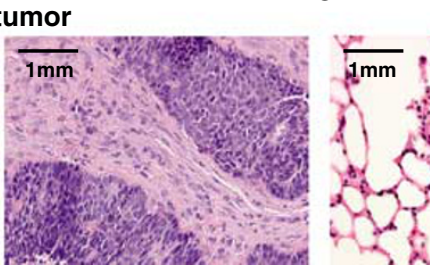

Lung

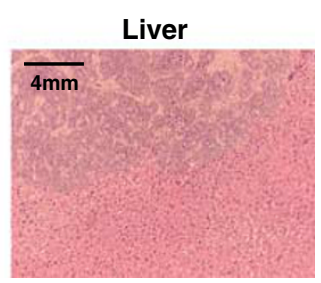

b

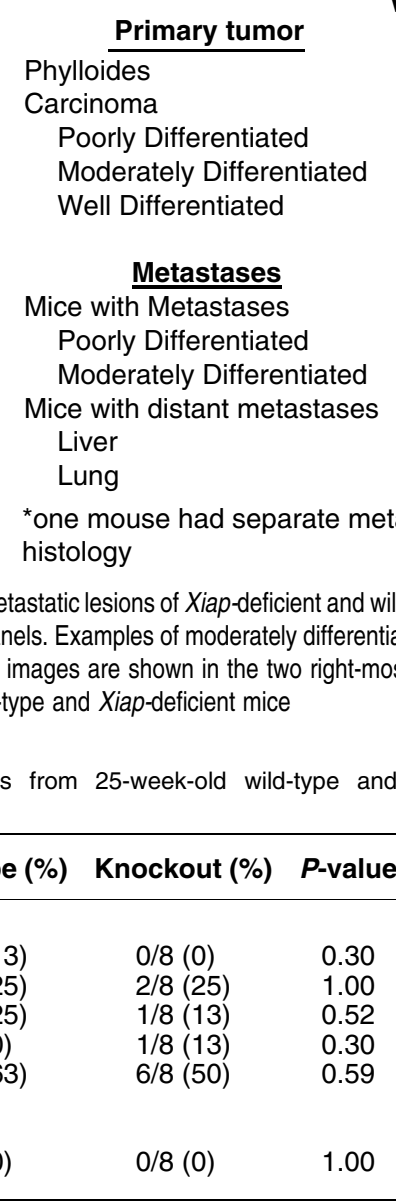

Wild type

$4 / 850 \%$

$4 / 8 \quad 50 \%$

$2 / 825 \%$

$1 / 813 \%$

$1 / 813 \%$

$5 / 8 \quad 63 \%$

$4 / 850 \%$

$1 / 813 \%$

$2 / 825 \%$

$1 / 813 \%$

$2 / 825 \%$

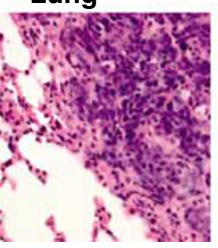

Knockout

\section{$3 / 933 \%$}

$6 / 9 \quad 67 \%$

$5 / 956 \%$

$1 / 9 \quad 11 \%$

$0 / 9 \quad 0 \%$

$5 / 9 \quad 56 \%$

$5 / 956 \%$

$1 / 911 \%$ *

$3 / 933 \%$

$3 / 933 \%$

$1 / 9 \quad 11 \%$
$\boldsymbol{P}$-value

0.49

0.49

0.20

0.87

0.27

0.77

0.82

0.93

0.71

0.31

0.45

*one mouse had separate metastases with poor and well-differentiated

Figure 4 Histology from primary and metastatic lesions of Xiap-deficient and wild-type TRAMP mice. (a) Examples of poorly differentiated tumors from wild-type and Xiapdeficient mice are shown in the left-most panels. Examples of moderately differentiated tumors are shown in the second set of panels. Metastases to liver and lung were also histologically confirmed and representative images are shown in the two right-most panels. (b) Results of histologic analysis for both primary and metastatic tumors were tabulated and compared between the wild-type and Xiap-deficient mice

Table 1 Histologic analysis of tumors from 25-week-old wild-type and Xiap-deficient TRAMP mice

\begin{tabular}{lccc}
\hline & Wild type (\%) & Knockout (\%) & $P$-value \\
\hline Primary tumor & & & \\
Phylloides & $1 / 8(13)$ & $0 / 8(0)$ & 0.30 \\
Carcinoma & $2 / 8(25)$ & $2 / 8(25)$ & 1.00 \\
$\quad$ Poorly differentiated & $2 / 8(25)$ & $1 / 8(13)$ & 0.52 \\
$\quad$ Well differentiated & $0 / 8(0)$ & $1 / 8(13)$ & 0.30 \\
PIN & $5 / 8(63)$ & $6 / 8(50)$ & 0.59 \\
Metastases & & & \\
Mice with metastases & $0 / 8(0)$ & $0 / 8(0)$ & 1.00 \\
\hline
\end{tabular}

Wild-type and Xiap-deficient mice were followed in cohorts of 10 mice until 25 weeks of age; eight of ten total mice were left in both groups at this time. Results from histologic analysis were tabulated and compared.

Finally, lysates from TRAMP tumors were assessed for expression of caspase- 9 and the presence of active caspase3 (Figure 7a). We did not detect any active caspase-3 in either wild-type or Xiap-deficient TRAMP tumors. This finding is consistent with our previous finding of generally low apoptotic rates in TRAMP prostate tumors.
Xiap deficiency does not increase c-IAP1 and c-IAP2 expression in TRAMP tumors. Of the mammalian IAP family members, only cellular IAP1 (c-IAP1) and c-IAP2 are capable of binding caspases and might functionally compensate in the apoptotic pathway following loss of XIAP. ${ }^{29}$ Furthermore, in the initial description of Xiapdeficient mice, it was found that c-IAP1 and c-IAP2 are overexpressed in Xiap-deficient mice. ${ }^{30}$ For this reason, expression of c-IAP1 and c-IAP2 was examined in prostate tumor specimens from control and Xiap-deficient mice by immunoblot (Figure 7a). There was no obvious compensatory overexpression of c-IAP1 or c-IAP2 in the Xiap-deficient TRAMP tumors. Detection of $\mathrm{c}-\mathrm{IAP} 1$ and c-IAP2 by immunoblot was quantified, and results are presented in Figure $7 \mathrm{~b}$. Not only was there no evidence of overexpression of C-IAP1 or C-IAP2, the mean level of C-IAP2 was actually decreased in the Xiap-deficient tumors compared to wild-type controls. Since levels of c-IAP1 and C-IAP2 are also subject to post-translational regulation, ${ }^{31}$ expression of $c$-iap1 and $c$-iap2 transcripts was also examined by quantitative RT-PCR. Consistent with measurements of protein expression, expression of $c$-iap1 
Xiap deficient

wild type
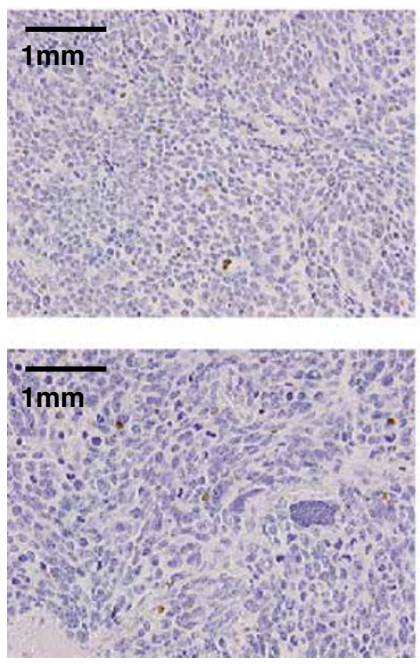

b

(1)

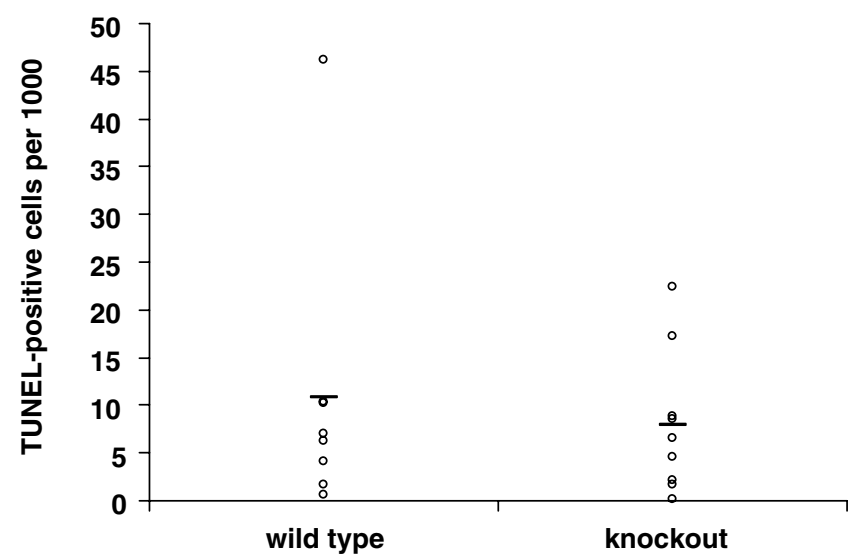

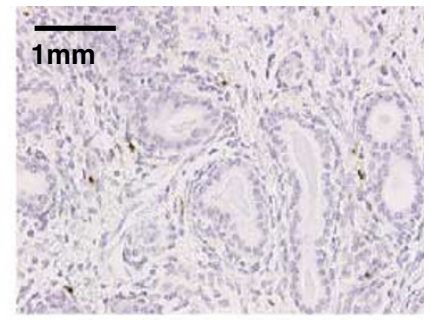

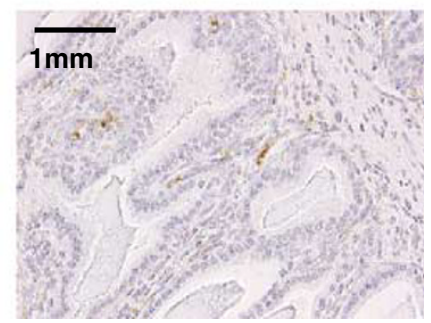

Figure 5 Frequency of apoptotic cells in Xiap-deficient tumors. (a) Primary prostate tumors were assessed for apoptotic cells using TUNEL staining. Representative images are shown from tumors with poorly differentiated carcinoma (left) and phylloides histology (right). (b) The number of TUNEL-positive cells per 1000 total cells was plotted for wild-type and Xiap-deficient mice. Mean values are represented by ( -$)$

and c-iap2 mRNA was not increased in Xiap-deficient tumors compared to controls (Figure $7 \mathrm{c}$ ). Levels of $c$-iap1 and $c$-iap2 transcripts were actually somewhat less in the Xiap-deficient group, which was surprising but correlated with our observations of protein levels. In summary, compensation by c-IAP1 or C-IAP2 could not explain why Xiap deficiency did not protect TRAMP mice against tumor onset, growth or lethality.

\section{Discussion}

Malignant cells demonstrate a resistance to apoptosis, which allows these cells to survive in cellular environments that would typically induce cell death. ${ }^{1}$ One proposed mechanism for the acquired capacity of cancer cells to evade apoptosis is antagonism of caspase activity through increased expression of XIAP. Elevated expression of XIAP has been demonstrated in cancers of various origins. ${ }^{10-16}$ Not only is XIAP overexpressed in cancer, increased XIAP expression has been shown to contribute to apoptosis resistance and conversely, XIAP antagonism sensitizes cancer cells to multiple types of apoptotic stimuli in vitro and in vivo. ${ }^{13,17-25,28,32-37}$ These apoptotic stimuli have included various chemotherapeutic agents, ionizing radiation, tumor necrosis factor-related apoptosis-inducing ligand, anoikis induction and immune clearance by cytotoxic lymphocytes. Despite these encouraging reports supporting a role for XIAP in the pathogenesis of cancer, there are data that are difficult to reconcile with the currently known functions of XIAP. Specifically, the paradoxical and dramatic favorable prognostic value of elevated XIAP levels observed in patients with resected prostate cancer does not correspond with the predicted increased resistance to apoptosis. ${ }^{12}$ Interestingly, C-IAP1 and c-IAP2 expression correlated negatively with prognosis in this same group of patients.

We report here that Xiap-deficient TRAMP mice do not develop tumors later than wild-type TRAMP mice. Furthermore, Xiap deficiency does not lessen the lethality, metastatic potential, histologic grade or tumor growth of TRAMP tumors; in fact, the data suggested a trend of more aggressive behavior in Xiap-deficient TRAMP tumors. Thus, we conclude that although elevations of XIAP expression have been 
a

\section{Xiap deficient}
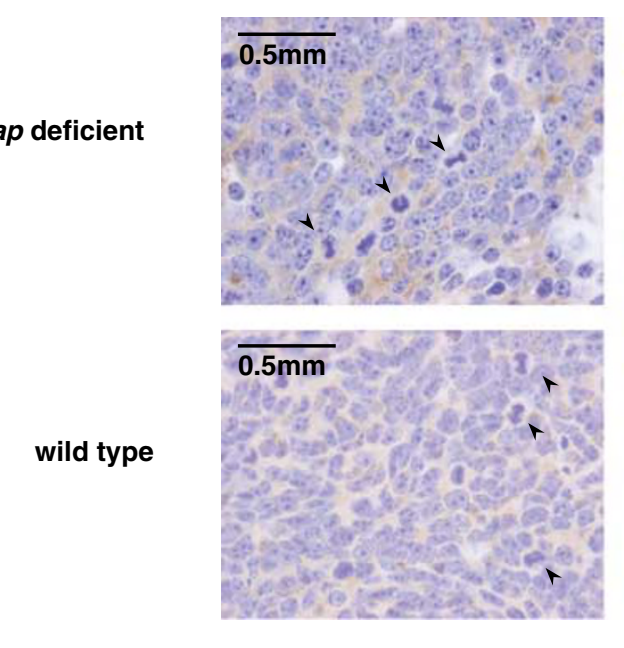

\section{Ki-67 staining}
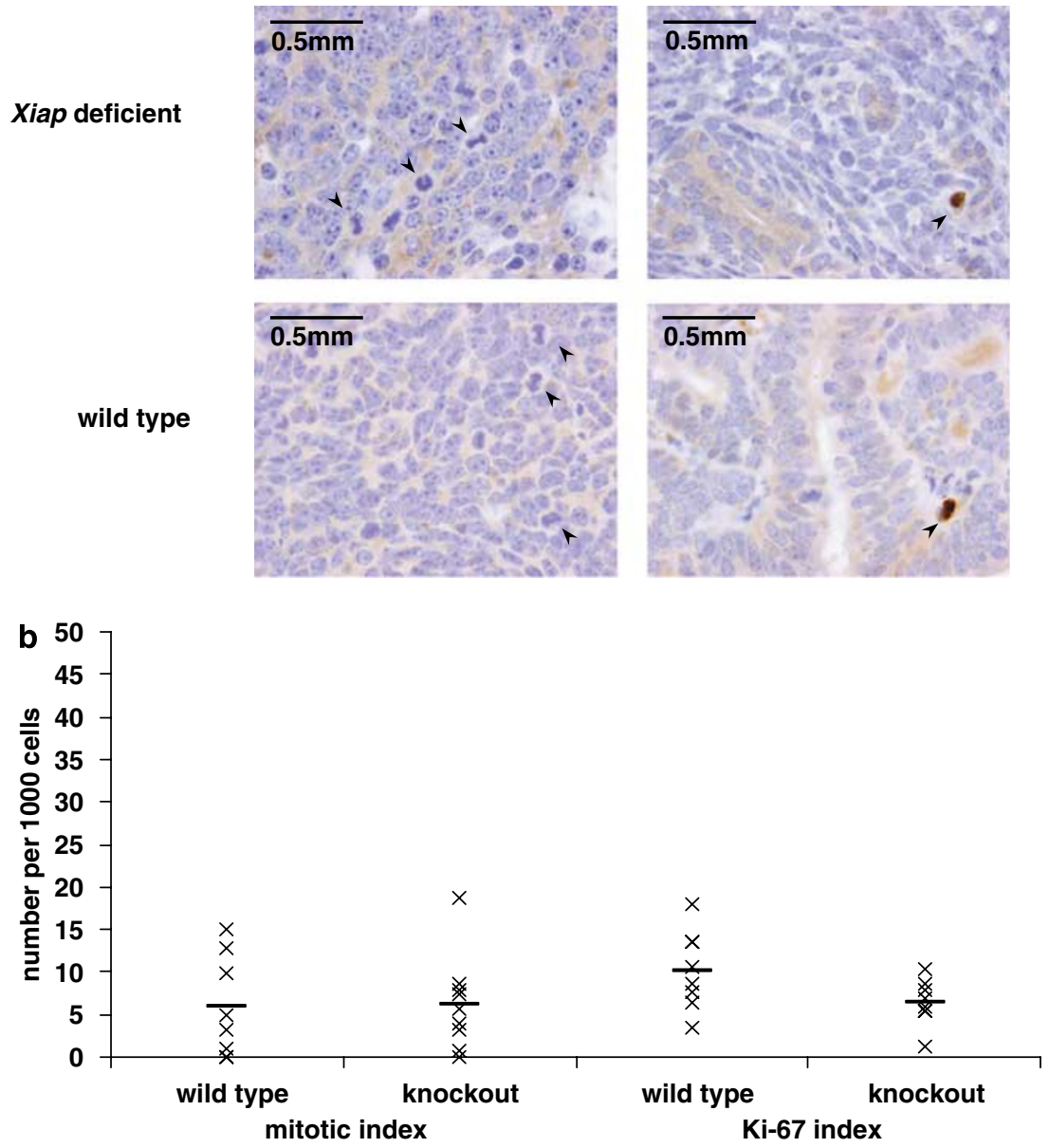

Figure 6 Proliferative indices in Xiap-deficient tumors. (a) Primary prostate tumors were stained immunohistochemically for Ki-67 (a proliferative marker). Ki-67 proliferative index and mitotic index were calculated. Representative images demonstrating both mitotic figures as well Ki-67-positive cells are shown in the left and right-hand panels, respectively. (b) The number of Ki-67-positive cells and the number of mitotic figures per 1000 total cells were plotted for wild-type and Xiap-deficient mice. Mean values are represented by $(-)$

observed in TRAMP tumors, upregulation of XIAP is not essential for transformation of prostate epithelium. It is possible that the aggressiveness of the TRAMP model, in which p53 and pRB are inhibited by the SV40 T-antigen, may have overwhelmed the ability to detect an effect of Xiap deficiency. In addition, the inhibition of p53 itself may be sufficient to inhibit apoptosis. However, the TRAMP model has been used to successfully establish a role for the antiapoptotic $\mathrm{BCl}-2$ in tumorigenesis, ${ }^{38}$ as well as to demonstrate that compounds such as green tea and celecoxib suppress tumorigenesis. ${ }^{39,40}$

These findings suggest that although XIAP is overexpressed in cancer it may not play a causal role in tumor pathogenesis. Conspicuously, evidence of XIAP mutations, translocations or amplifications, as is typically associated with classic oncogenes, has been absent in human cancers. Worth considering is the possibility that overexpression of XIAP may instead be a surrogate marker for other biologic behaviors. For example, XIAP is known to be upregulated by hypoxia ${ }^{32}$ and thus may be overexpressed in tumors that are outgrowing a vascular supply.
Alternatively, XIAP may modulate apoptosis and tumor progression without being a classic oncogene. In this case, tumor formation in the absence of XIAP could occur if increased apoptosis was compensated by an increase in proliferation. In fact, although Ferreira et al. ${ }^{26}$ did not observe a correlation between XIAP expression levels and apoptotic index in resected non-small cell lung cancer, they did note an increased proliferative and mitotic index in tumors with low XIAP expression. However, the surprising finding that Xiap deficiency did not result in an increase in the apoptotic index suggests that an increased proliferative index would not explain our findings. Indeed, when proliferative rate and mitotic index were assessed, there were no discernible differences between the wild-type and Xiapdeficient mice.

In addition, expression of the oncogenic SV40 T-antigen in the absence of XIAP may have selected for pathogenic mechanisms of apoptosis resistance that do not depend on $\mathrm{XIAP}$. This selection pressure may be less acute in a clinical setting when XIAP antagonists are given after cancer has already developed. To explore the possibility of 


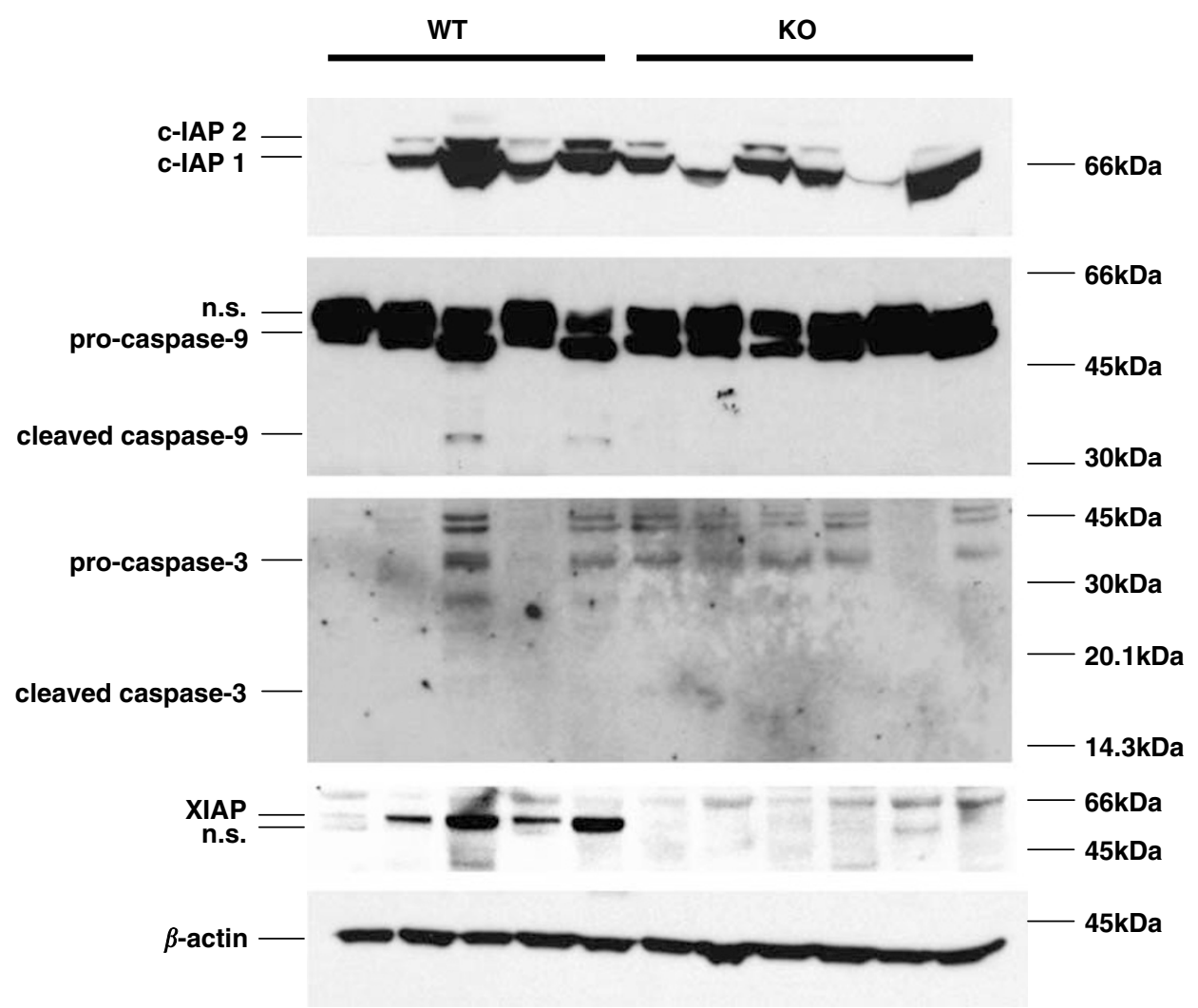

b

C-IAP1 protein quantitation

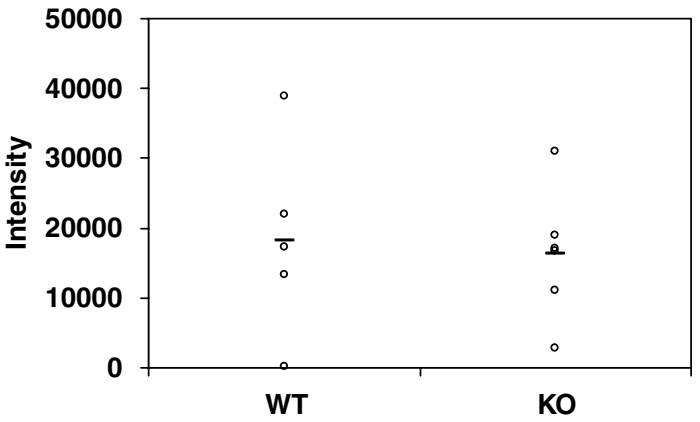

C

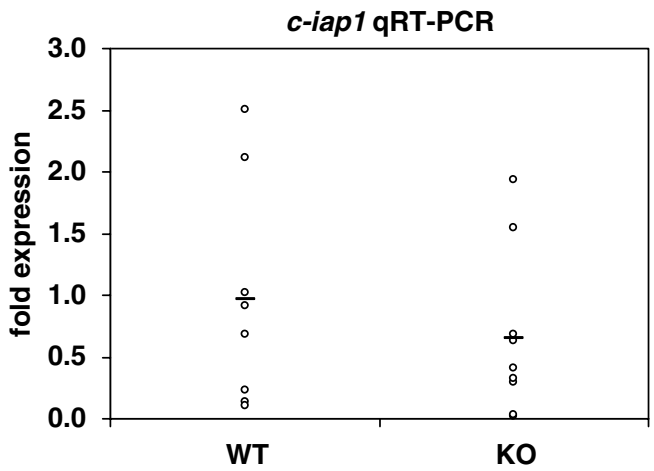

C-IAP2 protein quantitation
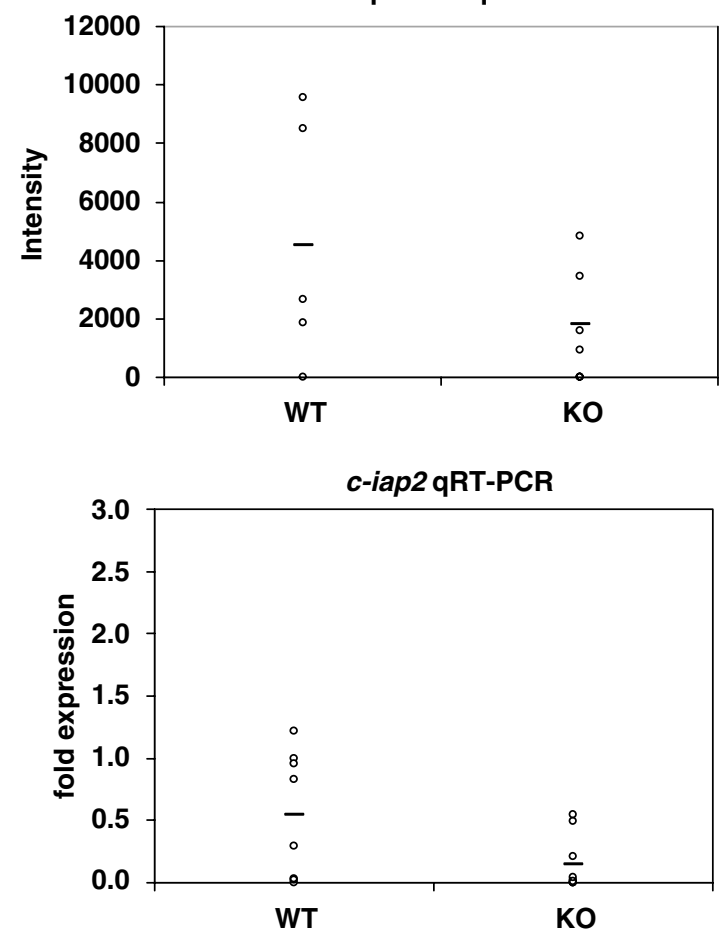

Figure 7 Expression of c-IAP1, C-IAP2 and other apoptotic proteins in Xiap-deficient TRAMP tumor specimens. (a) Immunoblot of c-IAP1 and c-IAP2 protein levels in primary tumor lysates. The same lysates were also probed for caspase-3 (full-length $32 \mathrm{kDa}$, cleaved form 19 or $17 \mathrm{kDa}$ ), caspase-9 (full-length $46 \mathrm{kDa}$, cleaved form $36 \mathrm{kDa}$ ) and XIAP ( $57 \mathrm{kDa})$. A $\beta$-actin immunoblot is shown to demonstrate equal total protein loading. (b) Expression levels of c-IAP1 and c-IAP2 protein based on immunoblotting were quantitated using ImageJ software. Relative intensity is plotted on the $y$ axis. Mean values are represented by (-). (c) Expression of $c$-iap1 and $c$-iap2 mRNA in tumor specimens, as assessed by quantitative RT-PCR. Fold expression relative to normal prostate is plotted on the $y$ axis for wild-type and Xiap-deficient cohorts. n.s. nonspecific 
XIAP-independent mechanisms of apoptosis inhibition, c-IAP1 and c-IAP2 were evaluated as obvious candidates for a possible compensatory effect. No evidence for such a role was found based on levels of C-IAP1 and C-IAP2 overexpression. Moreover, although c-IAP1 and C-IAP2 are capable of binding caspases, the affinity of C-IAP1 or C-IAP2 for caspases is much lower than XIAP, and recent data suggest that both are incapable of inhibiting caspase activity. ${ }^{29,41}$ If inhibition of caspases is relevant to the physiology of cancer cell biology and XIAP overexpression, it is unlikely that C-IAP1 or C-IAP2 can compensate for this activity.

Another possibility for our results is the absence of a significant apoptotic stimulus in our experiments. Although it has been reported that cancer cells can have high basal levels of apoptotic signaling molecules such as caspases-3 and $-8,{ }^{42}$ we did not observe significant apoptotic activity in TRAMP tumors. It is interesting that the active $35-\mathrm{kDa}$ form of caspase- 9 was observed only in the wild-type mice. However, there did not appear to be a significant amount of active caspase-3 detected in either wild-type or Xiap-deficient mice. This finding correlates with the relatively low apoptotic indices in these tumors as assessed by TUNEL staining. The absence of an effective apoptotic stimulus may have negated any effect from Xiap deficiency. Synergy between XIAP antagonism has been seen when combined with an apoptosis-inducing stimulus, even if little effect is seen in the absence of such a stimulus. Future combinations of chemotherapy or radiation therapy with XIAP antagonism in this or other transgenic cancer models may help resolve this possibility. XIAP antagonists may also have more clinical efficacy if combined with traditional cytotoxic therapies.

Given the promising preclinical reports of XIAP-targeted therapy, it is not surprising that these agents are being developed for clinical use. XIAP is a particularly attractive therapeutic target, because the apparent health of Xiapdeficient mice suggests that the side effects of suppressing XIAP should be minimal. The present study is, to our knowledge, the first evaluation of the role of XIAP in an immunocompetent autochthonous tumor model. Contrary to the previously discussed preclinical reports, we did not observe a protective effect in Xiap-deficient mice. Moreover, the suggestion of an adverse impact of Xiap deficiency on tumor progression, in addition to previous accounts of an adverse prognosis associated with low XIAP levels in prostate and lung cancer, underscores the need for a better understanding of the physiologic function of XIAP. These findings have obvious implications for treatments targeting XIAP activity. Neoplastic cells likely develop antiapoptotic mechanisms that do not depend on XIAP overexpression, which may have greater importance in the setting of XIAP antagonism. Greater insight into these pathways will help in selection of patients who would benefit most from XIAP-targeted therapies, as well as in overcoming resistance to such therapies.

\section{Materials and Methods}

Animals. Generation and genotyping of TRAMP and Xiap-deficient mice have been previously described. ${ }^{27,30}$ All mice were bred and maintained on a C57BL/6 background. TRAMP males were mated with $X_{i a p^{+1-}}$ females to generate Xiap TRAMP males, as well as Xiap ${ }^{+}$TRAMP males for controls. Research was conducted on a UCUCA-approved protocol in a manner consistent with the $\mathrm{NIH}$ Guidelines for the Care and Use of Laboratory Animals. Beginning at 12 weeks of age, weekly abdominal palpation by two independent observers was used to determine tumor onset. Mice were euthanized when moribund and samples were taken for histology, RNA and protein evaluation. The pelvic lymph nodes and the abdominal and thoracic cavities were observed for signs of metastasis and visible metastases were confirmed histologically.

MRI imaging. Mice were imaged with abdominal MRI biweekly by the Michigan Small Animal Imaging Resource (http://www.med.umich.edu/msair). Mice were anesthetized with $2 \%$ isoflurane-air mixture. Images were obtained with a $7.0 \mathrm{~T}$ Varian MR scanner (183-mm horizontal bore; Varian, Palo Alto, CA, USA). A double-tuned volume radiofrequency coil was used to scan the abdominal region of the mice. Axial $\mathrm{T}_{2}$-weighted images were acquired using a fast spin-echo sequence with the following parameters: repetition time/effective echo time, $4000 / 60 \mathrm{~ms}$; echo spacing, $15 \mathrm{~ms}$; number of echoes, 8; field of view $30 \mathrm{~mm} \times 30 \mathrm{~mm}$; matrix, $128 \times 128$; slice thickness, $1 \mathrm{~mm}$; slice spacing, $0.25 \mathrm{~mm}$; number of slices, 17 and number of scans, 4 (total scan time was approximately 4 min.).

Immunoblot. Previously frozen specimens were lysed on ice in radioimmunoprecipitation analysis buffer containing protease inhibitors. Membranes were probed using a 1:2000 dilution of rodent inhibitor of apoptosis protein (RIAP) rabbit polyclonal antibody (a gift from Peter Liston and Robert Korneluk), which recognizes mouse c-IAP1 and c-IAP2. Immunoblots were also probed for XIAP (goat polyclonal, R\&D, cat no. AF8221, Minneapolis, MN, USA), caspase-3 (rabbit polyclonal, a gift from Guy Salvesen) and caspase-9 (mouse monoclonal, Stressgen, cat no. AAM-139, Ann Arbor, MI, USA). $\beta$-Actin antibody was purchased from Sigma (St Louis, MO, USA). The following HRP-conjugated secondary antibodies were used: sheep anti-mouse (Amersham, Piscataway, NJ, USA), donkey anti-rabbit (Amersham) and donkey anti-goat (Serotec, Raleigh, NC, USA). Densitometry was performed by NIH ImageJ software (http:// rsb.info.nih.gov/ij/).

Quantitative RT-PCR. Tissue specimens were preserved in RNAlater (Ambion, Austin, TX, USA). RNA was extracted using an RNeasy kit (Qiagen, Valencia, CA, USA). CDNA was generated using RTScript reverse transcriptase and quantified using TaqMan gene expression assays (Applied Biosystems, Foster City, $\mathrm{CA}$, USA) on an $\mathrm{ABI} 7500 \mathrm{gPCR}$ instrument.

Histology. Hematoxylin and eosin-stained slides were prepared from paraffinembedded formalin-fixed tissue by the University of Michigan Tissue core. Slides were examined in a blinded fashion by a certified genitourinary pathologist (KJW) and classified as phylloides, PIN, or carcinoma. Carcinoma specimens were graded as well, moderately or poorly differentiated. Liver and lung sections were similarly inspected. TUNEL staining was performed with the Apoptag kit (Chemicon, Temecula, CA, USA) by the University of Michigan Tissue core. Digital images of five independent fields were taken of each tumor at $\times 40$ magnification. TUNELpositive cells were counted manually, while total cell number was determined by ImageJ analysis. Quantification was recorded as the number of TUNEL-positive cells per 1000 cells. Mitotic and proliferative indices were calculated in an analogous manner, although images were visualized at $\times 100$ to facilitate the identification of mitotic figures. Ki-67 staining was also performed by the University of Michigan Tissue core using a primary antibody from Dako (Carpinteria, CA, USA) and the MOM immunodetection kit from Vector Laboratories (Burlingame, CA, USA).

Statistical analysis. Statistical analysis for Kaplan-Meier plots was performed using a two-sided log-rank test. In addition, a one-sided log-rank test was used to determine whether the knockout mice had better outcomes than the wild-type mice. All other comparisons were performed using a two-tailed $\chi^{2}$ test. Calculations were performed using the R statistical computation system (http:// www.R-project.org). Values of $P<0.05$ were considered statistically significant.

Acknowledgements. We thank Drs. Peter Liston and Robert Korneluk for the generous gift of RIAP antibody, Dr. Guy Salvesen for his gift of caspase-3 antibody and Drs. Brian Ross and Brad Moffat for their assistance with MRI imaging. KO is a recipient of a Pre-doctoral Award funded through the Breast Cancer Research Program of the Department of Defense (W81XWH-06-1-0429). This study was supported by funding received from the Department of Defense 
(W81XWH-04-1-0891) and the National Institutes of Health (5R01GM067827-03). Dr. Colin Duckett serves as a consultant to Aegera Therapeutics and is a member of their scientific advisory board. All other authors have no conflicting interests.

1. Hanahan D, Weinberg RA. The hallmarks of cancer. Cell 2000; 100: 57-70.

2. Green DR, Evan GI. A matter of life and death. Cancer Cell 2002; 1: 19-30.

3. Nunez G, Benedict MA, Hu Y, Inohara N. Caspases: the proteases of the apoptotic pathway. Oncogene 1998; 17: 3237-3245.

4. Shiozaki EN, Shi Y. Caspases, IAPs and Smac/DIABLO: mechanisms from structural biology. Trends Biochem Sci 2004; 29 : 486-494.

5. Vaux DL, Silke J. IAPs, RINGs and ubiquitylation. Nat Rev Mol Cell Biol 2005; 6: 287-297.

6. Deveraux QL, Takahashi R, Salvesen GS, Reed JC. X-linked IAP is a direct inhibitor of celldeath proteases. Nature 1997; 388: 300-304.

7. LaCasse EC, Baird S, Korneluk RG, MacKenzie AE. The inhibitors of apoptosis (IAPs) and their emerging role in cancer. Oncogene 1998; 17: 3247-3259.

8. Holcik M, Gibson H, Korneluk RG. XIAP: apoptotic brake and promising therapeutic target. Apoptosis 2001; 6: 253-261.

9. Schimmer AD, Dalili S, Batey RA, Riedl SJ. Targeting XIAP for the treatment of malignancy. Cell Death Differ 2006; 13: 179-188.

10. Jaffer S, Orta L, Sunkara S, Sabo E, Burstein DE. Immunohistochemical detection of antiapoptotic protein X-linked inhibitor of apoptosis in mammary carcinoma. Hum Pathol 2007; 38: 864-870.

11. Kluger HM, McCarthy MM, Alvero AB, Sznol M, Ariyan S, Camp RL et al. The X-linked inhibitor of apoptosis protein (XIAP) is up-regulated in metastatic melanoma, and XIAP cleavage by phenoxodiol is associated with carboplatin sensitization. $J$ Transl Med 2007; 5: 6 .

12. Krajewska M, Krajewski S, Banares S, Huang X, Turner B, Bubendorf $L$ et al. Elevated expression of inhibitor of apoptosis proteins in prostate cancer. Clin Cancer Res 2003; 9 : 4914-4925.

13. Lopes RB, Gangeswaran R, McNeish IA, Wang Y, Lemoine NR. Expression of the IAP protein family is dysregulated in pancreatic cancer cells and is important for resistance to chemotherapy. Int J Cancer 2007; 120: 2344-2352.

14. Mizutani Y, Nakanishi H, Li YN, Matsubara H, Yamamoto K, Sato N et al. Overexpression of XIAP expression in renal cell carcinoma predicts a worse prognosis. Int J Oncol 2007; 30: 919-925.

15. Tamm I, Richter S, Oltersdorf D, Creutzig U, Harbott J, Scholz F et al. High expression levels of X-linked inhibitor of apoptosis protein and survivin correlate with poor overall survival in childhood de novo acute myeloid leukemia. Clin Cancer Res 2004; 10: $3737-3744$

16. Tamm I, Kornblau SM, Segall H, Krajewski S, Welsh K, Kitada S et al. Expression and prognostic significance of IAP-family genes in human cancers and myeloid leukemias. Clin Cancer Res 2000; 6: 1796-1803.

17. Berezovskaya O, Schimmer AD, Glinskii AB, Pinilla C, Hoffman RM, Reed JC et al. Increased expression of apoptosis inhibitor protein XIAP contributes to anoikis resistance of circulating human prostate cancer metastasis precursor cells. Cancer Res 2005; 65: 2378-2386.

18. Holcik M, Yeh C, Korneluk RG, Chow T. Translational upregulation of X-linked inhibitor of apoptosis (XIAP) increases resistance to radiation induced cell death. Oncogene 2000; 19: 4174-4177.

19. Liu Z, Li H, Derouet M, Filmus J, LaCasse EC, Korneluk RG et al. ras oncogene triggers up-regulation of CIAP2 and XIAP in intestinal epithelial cells: epidermal growth factor receptor-dependent and -independent mechanisms of ras-induced transformation. J Biol Chem 2005; 280: 37383-37392.

20. Liu Z, Li H, Wu X, Yoo BH, Yan SR, Stadnyk AW et al. Detachment-induced upregulation of XIAP and cIAP2 delays anoikis of intestinal epithelial cells. Oncogene 2006; 25: 7680-7690.

21. $\mathrm{Ng} \mathrm{CP}$, Bonavida B. X-linked inhibitor of apoptosis (XIAP) blocks Apo2 ligand/tumor necrosis factor-related apoptosis-inducing ligand-mediated apoptosis of prostate cancer cells in the presence of mitochondrial activation: sensitization by overexpression of second mitochondria-derived activator of caspase/direct IAP-binding protein with low pl (Smac/ DIABLO). Mol Cancer Ther 2002; 1: 1051-1058.
22. Nomura $T$, Mimata $H$, Takeuchi $Y$, Yamamoto $H$, Miyamoto $E$, Nomura $Y$. The $X$-linked inhibitor of apoptosis protein inhibits taxol-induced apoptosis in LNCaP cells. Urol Res 2003; 31: 37-44.

23. Amantana A, London CA, Iversen PL, Devi GR. X-linked inhibitor of apoptosis protein inhibition induces apoptosis and enhances chemotherapy sensitivity in human prostate cancer cells. Mol Cancer Ther 2004; 3: 699-707.

24. LaCasse EC, Cherton-Horvat GG, Hewitt KE, Jerome LJ, Morris SJ, Kandimalla ER et al. Preclinical characterization of AEG35156/GEM 640, a second-generation antisense oligonucleotide targeting X-linked inhibitor of apoptosis. Clin Cancer Res 2006; 12: 5231-5241.

25. McManus DC, Lefebvre CA, Cherton-Horvat G, St-Jean M, Kandimalla ER, Agrawal S et al. Loss of XIAP protein expression by RNAi and antisense approaches sensitizes cancer cells to functionally diverse chemotherapeutics. Oncogene 2004; 23: 8105-8117.

26. Ferreira CG, van der Valk P, Span SW, Ludwig I, Smit EF, Kruyt FA et al. Expression of $X$-linked inhibitor of apoptosis as a novel prognostic marker in radically resected non-small cell lung cancer patients. Clin Cancer Res 2001; 7: 2468-2474.

27. Greenberg NM, DeMayo F, Finegold MJ, Medina D, Tilley WD, Aspinall JO et al. Prostate cancer in a transgenic mouse. Proc Natl Acad Sci USA 1995; 92: 3439-3443.

28. Bilim V, Kasahara T, Hara N, Takahashi K, Tomita Y. Role of XIAP in the malignant phenotype of transitional cell cancer (TCC) and therapeutic activity of XIAP antisense oligonucleotides against multidrug-resistant TCC in vitro. Int J Cancer2003; 103: 29-37.

29. Eckelman BP, Salvesen GS. The human anti-apoptotic proteins CIAP1 and CIAP2 bind but do not inhibit caspases. J Biol Chem 2006; 281: 3254-3260.

30. Harlin H, Reffey SB, Duckett CS, Lindsten T, Thompson CB. Characterization of XIAP-deficient mice. Mol Cell Biol 2001; 21: 3604-3608.

31. Yang Y, Fang S, Jensen JP, Weissman AM, Ashwell JD. Ubiquitin protein ligase activity of IAPs and their degradation in proteasomes in response to apoptotic stimuli. Science 2000; 288: 874-877.

32. Marienfeld C, Yamagiwa Y, Ueno Y, Chiasson V, Brooks L, Meng F et al. Translational regulation of XIAP expression and cell survival during hypoxia in human cholangiocarcinoma. Gastroenterology 2004; 127: 1787-1797.

33. Cummins JM, Kohli M, Rago C, Kinzler KW, Vogelstein B, Bunz F. X-linked inhibitor of apoptosis protein (XIAP) is a nonredundant modulator of tumor necrosis factor-related apoptosis-inducing ligand (TRAIL)-mediated apoptosis in human cancer cells. Cancer Res 2004; 64: 3006-3008.

34. Ravi R, Fuchs EJ, Jain A, Pham V, Yoshimura K, Prouser T et al. Resistance of cancers to immunologic cytotoxicity and adoptive immunotherapy via $X$-linked inhibitor of apoptosis protein expression and coexisting defects in mitochondrial death signaling. Cancer Res 2006; 66: 1730-1739.

35. Arnt CR, Chiorean MV, Heldebrant MP, Gores GJ, Kaufmann SH. Synthetic Smac/DIABLO peptides enhance the effects of chemotherapeutic agents by binding XIAP and CIAP1 in situ. J Biol Chem 2002; 277: 44236-44243.

36. Sasaki H, Sheng Y, Kotsuji F, Tsang BK. Down-regulation of X-linked inhibitor of apoptosis protein induces apoptosis in chemoresistant human ovarian cancer cells. Cancer Res 2000; 60: 5659-5666.

37. Schimmer AD, Welsh K, Pinilla C, Wang Z, Krajewska M, Bonneau MJ et al. Smallmolecule antagonists of apoptosis suppressor XIAP exhibit broad antitumor activity. Cancer Cell 2004; 5: 25-35.

38. Bruckheimer EM, Brisbay S, Johnson DJ, Gingrich JR, Greenberg N, McDonnell TJ. Bcl-2 accelerates multistep prostate carcinogenesis in vivo. Oncogene 2000; 19: 5251-5258.

39. Gupta S, Hastak K, Ahmad N, Lewin JS, Mukhtar H. Inhibition of prostate carcinogenesis in TRAMP mice by oral infusion of green tea polyphenols. Proc Natl Acad Sci USA 2001; 98: 10350-10355.

40. Gupta S, Adhami VM, Subbarayan M, MacLennan GT, Lewin JS, Hafeli UO et al Suppression of prostate carcinogenesis by dietary supplementation of celecoxib in transgenic adenocarcinoma of the mouse prostate model. Cancer Res 2004; 64 3334-3343.

41. Tenev $T$, Zachariou $A$, Wilson R, Ditzel M, Meier P. IAPs are functionally non-equivalent and regulate effector caspases through distinct mechanisms. Nat Cell Biol 2005; 7: 70-77.

42. Yang L, Cao Z, Yan H, Wood WC. Coexistence of high levels of apoptotic signaling and inhibitor of apoptosis proteins in human tumor cells: implication for cancer specific therapy. Cancer Res 2003; 63: 6815-6824 